\title{
A Review of Non-Invasive Methods of Monitoring Intracranial Pressure
}

\author{
Derek Pobi Asiedua ${ }^{a}$ Kyoung-Jae Lee ${ }^{\mathrm{b}, \mathrm{d}}$, Godfrey Mills ${ }^{\mathrm{a}}$, Elsie Effah Kaufmann ${ }^{\mathrm{c}}$
}

\begin{abstract}
Intracranial pressure (ICP) monitoring is an important aspect of neuro-medicine. ICP is the pressure created by the presence of cerebrospinal fluid. ICP monitoring techniques consist of invasive (in vivo) and non-invasive methods (in vitro). Modern research aims to eliminate invasive monitoring of ICP and promote non-invasive methods of monitoring ICP. This review aims to assess the current methods and research related to non-invasive monitoring of ICP. Invasive methods of monitoring ICP remain the most accurate methods of measuring ICP but are associated with many complications. This review discusses the current approaches, advantages and disadvantages of these approaches in ICP monitoring. A critical study of the literature review demonstrates that for accuracy and precision, an innovative non-invasive method for ICP monitoring is needed. There is more room for further research and development in ICP monitoring. A universal method of ICP monitoring is needed and not for a class or group of patients.
\end{abstract}

Keywords: Review; Non-invasive; Intracranial pressure; Monitoring

\section{Introduction}

The brain consists of three main parts: the brain tissue (BT), cerebrospinal fluid (CSF) and the cerebral blood flow (CBF)

\footnotetext{
Manuscript accepted for publication February 27, 2014

${ }^{a}$ Computer Engineering Department, Faculty of Engineering, University of Ghana, Lagon Boundary, Accra, Legon, Ghana

${ }^{b}$ Department of Electronics and Control Engineering, College of Information Technology, Hanbat National University, 125,

Dongseo-daero, Yuseong-gu, Daejeon, Korea

${ }^{c}$ Biomedical Engineering Department, Faculty of Engineering, University of Ghana, Lagon Boundary, Accra, Legon, Ghana

${ }^{\mathrm{d}}$ Corresponding author: Kyoung-Jae Lee, Department of Electronics and Control Engineering, College of Information Technology, Hanbat

National University, 125, Dongseo-daero, Yuseong-gu, Daejeon, Korea.

Email: kyoungjae@hanbat.ac.kr
}

doi: http://dx.doi.org/10.14740/jnr269w
[1]. The intracranial pressure (ICP) is regarded as the cerebral fluid pressure. Increased ICP development may be acute or chronic [1]. The increase in ICP is usually caused by intracranial tumors, blood vessel anomalies and infections [1]. ICP measurement is an extremely important part of the neurosurgical armamentarium [1]. A few methods for measuring ICP are in existence and are being practiced in the health sector. These can be grouped into invasive and non-invasive methods [2]. The invasive methods involve penetrating the human body to attain the ICP [2], whereas the non-invasive methods do not involve penetrating the human body [2]. Some of the invasive methods involve the use of lumbar puncture, ventricular catheter or an external transducer. Fontanometer, transcranial Doppler (TCD) sonography, radiographic findings, ultrasound and infrared pupillometer are examples of non-invasive methods for measuring ICP [3]. Now, research into techniques for measurement of ICP is gearing towards non-invasive methods in a bid to eliminate complications associated with invasive methods [3].

Several reviews of work being done in the area of ICP monitoring have been done by various individuals. North's review of ICP monitoring discussed the importance of ICP monitoring, the transition from lumbar puncture through ventricular puncture to the combination of ventricular catheter and external transducers (that is due to the introduction of the strain gauge) [4]. He went further to talk about the various methods, devices and their advantages and disadvantages, and the interpretation of ICP monitoring [4]. His study only covered invasive methods of ICP monitoring. He concluded that the catheter tip transducer and the implanted microchip transducers are the best methods and gold standards of monitoring ICP [4]. Zhong et al's review concentrated on ICP devices and available research that had been done in the relevant field between 1977 and 2002 [2]. Their work was also geared towards finding the best method for monitoring ICP. Zhong et al's work focused on a classification of ICP monitoring based on the technology applied and the position of ICP measurement [2]. They further categorized research work done into in vitro tests, animal studies, pediatric research, mathematical/computer models and indirect measurements. They further discussed the complications, and the accuracy and stability of the various methods [2]. Zhong 
Table 1. A Summary on Existing Invasive ICP Monitoring Methods From Reviews

\begin{tabular}{ll} 
Device & Advantage \\
& \\
\hline Intraventricular catheter & 1. Gold standard of accuracy. \\
& 2. Allows drainage and sampling of CSF. \\
& 3. Allows ICP control. \\
4. & Inexpensive.
\end{tabular}

Subarachnoid bolt/screw

1. Quickly and easily placed.

2. Does not invade brain.

3. Allows sampling of CSF.

4. May have lower infection rate.

Subdural, epidural

catheter/sensor

Fiberoptic probe/catheter tip strain gauge

Telemetry operation
1. Least invasive.

2. Easily and quickly placed.

1. Can be placed in the subdural, subarachnoid, intraventricular or intraparenchymal spaces.

2. Easily transported.

3. Minimal artefact and drift.

4. High resolution of waveform.

5. No irrigation less risk of infection.

6. No need to adjust for patient position.

1. It supports short-term measurements.

2. It is easy to implement.

3. Allows larger range of use (home and medical center).
Disadvantages

1. Most invasive.

2. Sometimes difficult to cannulate ventricle.

3. Catheter can be occluded by blood or tissue.

4. Needs reposting of transducer level with change in head position.

5. Potential infection.

1. Blocked by swollen brain.

2. Catheter can be occluded by tissue or blood.

3. Must be balanced and recalibrated frequently.

1. Increasing baseline drift over time, accuracy and reliability are questionable.

2. Does not provide CSF sampling.

1. Cannot be recalibrated after it is placed, unless a ventriculostomy is used simultaneously for reference.

2. Breakage of the fiberoptic cable.

3. High cost.

1. Possibility of bacteria transmission.

2. High cost.

3. Infections and complications. et al then concluded that the ventricular catheter connected to an external strain gauge transducer or catheter tip pressure transducer device is the most accurate method of monitoring ICP [2]. Also, frequent external calibration of new devices (miniaturized transducers, fiberoptic systems) during patient monitoring should be done. Rosenberg et al's review concentrated on non-invasive methods of monitoring ICP [2]. Rosenberg et al classified the non-invasive methods by the theory or method used in monitoring ICP [3]. The categories consisted of physical examination, radiologic findings, TCD sonography, audiological techniques and near-infrared spectroscopy (NIRS) [3]. These methods were discussed into detail and a conclusion was made that ultrasound measurement of the optic nerve sheath diameter and Doppler flow are the most promising non-invasive methods for ICP monitoring [3]. A summary of the reviewers' comments on the pros and cons of both invasive and non-invasive methods of ICP monitoring can be seen in Table 1 and Table 2 respectively.

Jackrit et al also performed a review on ICP monitor- ing methods complimenting and referencing Zhong et al's review $[2,5]$. Their review covered both invasive and noninvasive methods of ICP monitoring. The main categories were [5]: 1) traditional methods of ICP; monitoring and measurement; 2) non-invasive methods for ICP monitoring; 3) developing methods of ICP monitoring.

Their review talked about the various methods, their advantages and disadvantage. Unlike their predecessors (above reviews), they did not single out one method as the most promising non-invasive method nor did they conclude their review [5]. All the reviews discussed into details the advantages and disadvantages of invasive and non-invasive ICP monitoring techniques [2-5]. It can be seen from various literature that invasive ICP still stands as the most accurate method for ICP monitoring as stated by Zhong et al and North [2, 4]. Current research looks to eliminate invasive ICP monitoring and eradicate the complications associated with it. This article seeks to critically review the current emerging trends in non-invasive ICP monitoring methods 
Table 2. A Summary on Existing Non-Invasive ICP Monitoring Methods From Reviews

\begin{tabular}{|c|c|c|}
\hline Technique & Advantages & Disadvantages \\
\hline Modeling & $\begin{array}{l}\text { 1. Easy to implement. } \\
\text { 2. Eliminates problems associated with invasive methods } \\
\text { (complications, infections and discomfort). } \\
\text { 3. Continuous measurement achievable. }\end{array}$ & $\begin{array}{l}\text { 1. Accuracy issues. } \\
\text { 2. Lack of substantial database. } \\
\text { 3. Issues with measuring physiological } \\
\text { parameters. } \\
\text { 4. Clinically not applicable. }\end{array}$ \\
\hline Medical imaging & $\begin{array}{l}\text { 1. Easy to implement. } \\
\text { 2. Eliminates problems associated with invasive methods } \\
\text { (complications, infections and discomfort). } \\
\text { 3. Clinically applicable. }\end{array}$ & $\begin{array}{l}\text { 1. Accuracy issues. } \\
\text { 2. Continues measurement not achieva }\end{array}$ \\
\hline Alternative methods & $\begin{array}{l}\text { 1. Easy to implement. } \\
\text { 2. Eliminates problems associated with invasive methods } \\
\text { (complications, infections and discomfort). } \\
\text { 3. Continuous measurement achievable. } \\
\text { 4. Clinically applicable. }\end{array}$ & $\begin{array}{l}\text { 1. Accuracy issues. } \\
\text { 2. Indirect methods of measurement. }\end{array}$ \\
\hline
\end{tabular}

being researched on, their pros and cons and give a conclusion on the bases of what the future holds for non-invasive ICP monitoring.

\section{Non-Invasive ICP Measurement}

Numerous investigations have shown that sustained intracranial hypertension is associated with poor diagnosis [2, 3]. Most importantly, the risk of intracranial infection, pain, discomfort, reliability and accuracy are problems associated with the existing invasive methods of measuring ICP $[2,3]$. Also, accuracy and reliability are problems associated with existing non-invasive methods of measuring ICP [3, 5]. Therefore, continuous monitoring, complications, discomfort and accuracy are problems associated with current methods for measuring ICP. From the four reviews stated above, developing techniques for measurement of ICP can be grouped into mathematical models, computational models, medical imaging and alternative techniques [2-5]. Even though the existing non-invasive methods of measuring ICP eliminate most of the problems associated with the invasive methods, these methods also have major problems associated with them. The current methods reviewed in this section of this paper are summarized in Supplementary 1 (www. neurores.org).

\section{Computational methods}

The model-based non-invasive estimation of ICP from cere- bral blood flow velocity (CBFV) and arterial pressure by Kashif et al uses routinely acquired signals with a physiological model to generate patient specific estimates of ICP [6]. The model consists of a small number of physiological parameters, therefore reducing the computation time but limiting its real-time predictions [7]. This is because the signals (arterial blood pressure and CBFV) have to first be detected and then serve as inputs to the physiological model [6, 7]. With this process, there is a short but "significant delay" which may cause complications in critical condition patients [7]. Kashif et al also mentioned a few constraints of their ICP monitoring method, which include the need for high sampling rate, the use of archived data and not current or real-time data and the possibility of face shifting measured [7]. With their discussed method continuing, calibration free and patient specific estimation of ICP was achieved [6]. Further work to optimize the proposed method was suggested by Kashif et al. The optimization will reduce computation time $[6,7]$ but the development of a more complex model will rather reduce the computation time [7].

Budohoski et al's work on assessing the accuracy of non-invasive estimation of ICP and its three main components (amplitude of ICP pulse, amplitude of the respiratory component and amplitude of the ICP slow waves) is discussed next [8]. This method is a convenient way for measuring ICP in wards as stated in the literature. The method is time-consuming and needs computation of PI [7, 8]. Medical staff and paramedics can perform this simple test with acceptable reliability. The deficiency of this method is its range of application. It can only be used in no other place but in 
wards. However, the absolute values of PI may not be used to predict the ICP since various physiological and disease states can produce similar and false positive TCD finding range [8].

Validation of CSF pressure measured, unattainable bedside monitoring in ICU, calibration/tuning need for each patient due to assumptions made and the assumption that RVP is constant are the limitations associated with Golzan et al's proposed methodology [9]. Further research on this method can be performed to optimize the algorithm but may also encounter an elongation in computation time [9]. Unlike Kashif et al, this may need tuning for each patient. Also, patients with eye problems (disease) cannot be monitored with this method making it patient specific (namely for a specific group of patients) [6,9]. Kim et al applied data mining technique on finding ICP [10]. This involved using data acquired from arterial blood pressure, CBFV and ICP. Like the above listed computational model, more data mining algorithms can be employed in further research to make the model better but this will consume much more time and it is also expensive to implement [10]. From Kashif et al, mapping may be associated with certain issues of adequate and robust capturing of parameters to predict ICP [6]. Kashif et al also stated that it is also expensive to perform such a monitoring technique in terms of hardware, computation and human resource. From Kashif et al and the review on Kashif et al's research, the computational time and real-time assessment must be worked on even though accuracy holds a higher priority $[6,11]$.

\section{Developed devices}

Bartusis et al's work on "Ophthalmic artery (OA) as a sensor for non-invasive intracranial pressure measurement electronic system" aimed at finding a measurement method which works perfectly without any calibration and a principle that could be applied for a non-invasive ICP meter [12]. Gravimetric scaling principle was used to compare OA pressure and an external pressure source [12]. The author places the TCD probe precariously close to the lens [12]. Their method would only be applicable in alert and cooperative individuals but this may not be possible with unconscious patients [12]. On the other hand, the accurate measurement of ICP was achieved with their method and principle but limited application to a group of patients (namely only conscious humans). In addition, prolonged pressure application to the globe can reduce central retinal artery blood flow and may result in vision complications $[11,12]$. The method can also not be used for continuous measurement due to the application of pressure on the eye. A long-term use may lead to total damage of the eye. It can also be stated that real-time acquisition of ICP cannot be achieved with this method. Also, the assumption or theory that OA exists in two segments is debatable. A review of the authors' research by Liebeskind et al states that microanatomical studies have shown different configurations and variability reporting that incidence of the OA originating from the intradural portion of the internal carotid artery ranges from $4.7 \%$ to $89.6 \%$ of individuals [7]. This also proves that this technique for measuring ICP is limited to a specific population.

Using the principle of impedance mismatch, Swoboda et al calculated ICP non-invasively [13]. The device developed and patented by Swoboda et al consists of a pressure sensor, analyzer and a display [13]. With this novel device, bedside monitoring can be achieved and the device could be used on all kinds of patients. Some issues exist in Swoboda et al's proposed ICP monitoring device. Just like other discussed methods listed in the computational model section, Swoboda et al developed an algorithm for the analyzer after feature extraction [13]. With provision of real-time data from the pressure sensor, the next most important step is the processing speed and time. An already acquired algorithm is placed into the device. Ji et al also developed an instrument using multiparameter fusion methods to calculate ICP [14]. Their device consists of a virtual device which does the multi-parameter fusion method with parameters acquired from a TCD instrument and FVEP [13]. With this, the device is not only bulky and complex but needs at least two healthcare personnel to operate it.

\section{Other methods}

Xie et al presented a non-invasive ICP estimation method depending on orbital subarachnoid space width (OSESW) measurement as inputs [15]. Their research is based on the theory that: 1) The pressure in the orbital subarachnoid space is in correlation with ICP [15]; 2) There exists a linear relation between the optic nerve sheath diameter and lumbar CSF pressure [15].

The method proposed by Xie et al cannot be used for continuous monitoring of ICP. The need to first acquire the OSESW before estimating ICP is not only time-consuming but also causes harm and discomfort to patients [15]. The patient condition can also affect the usage of this method since intensive care, that is, the MRI unit cannot be placed in the ICU and bedside monitoring cannot be achieved.

Weerakkody et al's article talked about studying the synchronization between ICP and NIRS variables induced by vasogenic waves of ICP during an infusion study in hydrocephalus patients and after traumatic brain injury [16]. The theories used in the research were: 1) "Slow waves in intracranial pressure occurring in the low frequency band are present across a wide range of conditions (illness)" [16]; 2) "NIRS allows detection of changes in brain tissue, cerebral blood volume (CBV) and $\mathrm{CBF}$ by recording relative changes in the concentration of $\mathrm{Hb}$ and $\mathrm{HbO}_{2}$ during cyclic hemodynamic changes associated with slow ventilation" [16].

Computerized infusion test is a well-established method 
for assessing CSF dynamics [16]. NIRS is also a well-established method for assessing tissue oxygenation index. Therefore, the data collection process using the study protocol was without fault [16]. The theory of using NIRS to detect changes in $\mathrm{BT}, \mathrm{CBV}$ and $\mathrm{CBF}$ by using changes recorded in the concentration of $\mathrm{Hb}$ and $\mathrm{HbO}_{2}$ was also accurate. The theory of slow waves in ICP occurs across a wide range of health conditions and can also be found in healthy individuals $[7,16]$. Therefore, this proposed method can only be used on patients with known conditions to monitor their ICP. The theory of small waves being present across a range of conditions can also be found in normal individuals $[7,16]$. The researcher believes that the correlation between ICP and NIRS has not been well established from their conclusion. Also, changes in concentration of $\mathrm{Hb}$ and $\mathrm{HbO}_{2}$ being a potential marker for strong vasogenic activities in ICP are debatable $[7,16]$.

\section{Conclusion}

The health and wellbeing of each patient is a doctor's desire. Comfort and healing is the desire of each and every patient in a healthcare center. There is possibility of acquiring comfort and prevention of complications associated with current ICP monitoring methods used in our healthcare centers. Patients and healthcare providers' needs have inspired further research into non-invasive methods of ICP monitoring. Many innovative and novel methods of non-invasive ICP monitoring have been developed over the last decade. It can be seen from the vast number of publications that researchers have not given up on finding the ideal ICP monitoring device. Research on non-invasive ICP monitoring will continue until a device is realized that: 1) measures ICP accurately; 2) eradicates the complications of invasive ICP monitoring; 3 ) achieves real-time and continuous ICP monitoring.

Further research into ICP monitoring will be facilitated by new and cutting edge technologies (such as MEMS, VLSI, wireless techniques, AI and many others). Research into this area will be concentrated on developing new or improving ICP sensor method, development and optimization of signal processing techniques, and identification, development and optimization of data mining and machine learning. The successful development of such a device will therefore contribute to producing a reference point for manufacturers in design ICP measuring equipment for use in the healthcare sector. Above all, the device developed will rival existing devices for ICP measuring in the healthcare sector.

\section{Acknowledgement}

This research is supported by University of Ghana and the Carnegie Corporation of New York for their support through the UG-Carnegie Next Generation of Academics in Africa Scholarship, and the research fund of Hanbat National University in 2013 .

\section{Source of Support}

UG-Carnegie Next Generation of Academics in Africa Scholarship and the research fund of Hanbat National University in 2013.

\section{Conflict of Interest}

The authors declare they have no conflict of interests.

\section{References}

1. Kashif FM, Heldt T, Verghese GC. Model-Based Estimation of Intracranial Pressure and Cerebrovascular Autoregulation. Massachusetts Institute of Technology, Computers in Cardiology. 2008;35:369-372.

2. Zhong J, Dujovny M, Park HK, Perez E, Perlin AR, Diaz FG. Advances in ICP monitoring techniques. Neurol Res. 2003;25(4):339-350.

3. Rosenberg JB, Shiloh AL, Savel RH, Eisen LA. Noninvasive methods of estimating intracranial pressure. Neurocrit Care. 2011;15(3):599-608.

4. North B. Intracranial pressure monitoring (chapt 10). In Reilly P, Bullack R (eds). Head injury, 2nd edition, London, UK:Hodder Arnold, 2005, pp. 209-216.

5. Jackrit S, Sattayasoonthorn P. A Review of Development for Intracranial Pressure Measurement. In Proceedings of the 7th Asian Conference on Computer-Aided Surgery (ACCAS 2011). Bangkok, Thailand, 2011.

6. Kashif FM, Verghese GC, Novak V, Czosnyka M, Heldt T. Model-based noninvasive estimation of intracranial pressure from cerebral blood flow velocity and arterial pressure. Sci Transl Med. 2012;4(129):129ra144.

7. Griggs RC. WriteClick Editor's Choice. American Academy of Neurology. 2013;507-508.

8. Budohoski PK, Schnidt B, Smielewski P, Kasprowicz M, Plantke R, Pickard JD, et al. Non-invasive estimated ICP pulse amplitude strongly correlates with outcome after traumatic Brain Injury. Acta Neurochir. Suppl. (Wien). 2012, Intracranial Pressure and Brain Monitoring XIV, pp. 121-124.

9. Golzan SM, Kim MO, Seddighi AS, Avolio A, Graham SL. Non-invasive estimation of cerebrospinal fluid pressure waveforms by means of retinal venous pulsatility and central aortic blood pressure. Ann Biomed Eng. 2012;40(9):1940-1948.

10. Kim S, Scalzo F, Bergsneider M, Vespa P, Martin N, Hu 
$X$. Noninvasive intracranial pressure assessment based on a data-mining approach using a nonlinear mapping function. IEEE Trans Biomed Eng. 2012;59(3):619-626.

11. Goldstein B, Tasker RC, Wakeland W. From Lundberg to SIM-ICP: computational physiology and modeling intracranial pressure. Sci Transl Med. 2012;4(129):129fs 126.

12. Bartusis R, ZXakelis R, Daubaris G, Ragauskas A. Ophthalmic Artery as a sensor for non-invasive intracranial pressure measurement electronic system. 2012;122(6):45 -48.

13. Swoboda M, Hochman GM, Fritz FJ. Non-invaqsiev intracranial pressure sensor. US, 2913/0289422.

14. Ji Z, Peng C. A New Noninvasive Intracranial Pressure
Measurement Instrument Based upon Multi-parameters Fusion Methods. in ICBEB '12 Proceedings of the 2012, 2012, pp. 666-669.

15. Xie X, Zhong X, Wang H, Jonas BJ, Peng X, Tian G, Xian J, et al. Non-invasive intracranial pressure estimation by orbital subarachnoid space measurement: the Beijing Intracranial and Intraocular Pressure (iCOP) study. Crit Care. 2013;17(4):162.

16. Weerakkody RA, Czosnyka M, Zweifel C, Castellani G, Smielewski P, Brady K, et al. Near infra-red Spectroscopy as Possible Non-invsasive Monitor of slow vasogenic ICP waves. Acta Neurochir. Suppl. (Wien). Intracranial Pressure and Brain Monitoring XIV, 2012, pp. 181-184. 